\title{
Diffuse soft tissue emphysema as a complication of anorexia nervosa
}

\author{
AI Hatzitolios, ML Sion, AD Kounanis, EN Toulis, A Dimitriadis, I Ioannidis, GN Ziakas
}

\begin{abstract}
Summary
We describe a case of a young woman with anorexia nervosa with a $35 \%$ weight loss, amenorrhea and behavioural disturbances complicated by spontaneous diffuse soft tissue emphysema (subcutaneous, pneumomediastinum, epidural and retroperitoneal).
\end{abstract}

Keywords: anorexia nervosa, soft tissue emphysema

Anorexia nervosa is a primary behavioural disorder characterised by a disturbed sense of body image, a morbid fear of gaining weight, disturbed eating pattern, marked weight loss and amenorrhea. It usually affects adolescent women $(95 \%) .^{1}$ Medical complications of anorexia nervosa can involve all organic systems of the body. ${ }^{2,3} \mathrm{~A}$ rare complication is spontaneous pneumomediastinum, with only a few cases reported worldwide. ${ }^{4}$ We report a case of anorexia nervosa complicated by diffuse soft tissue emphysema, pneumomediastinum and subcutaneous, retroperitoneal and epidural emphysema. Such findings have, to our knowledge, only been reported once in association with anorexia nervosa. ${ }^{5}$

\section{Case report}

A 15-year-old schoolgirl was admitted with a four-day history of a sore throat and retrosternal thoracic pain. The pain radiated to the shoulders and back, and intensified on swallowing and movement. No previous episodes of acute chest pain or violent coughing were mentioned. She was extremely cachectic (34 kg with a height of $166 \mathrm{~cm}$ ) and reported weight loss of $18 \mathrm{~kg}$ ( $35 \%$ of her normal body weight) in the previous six months and secondary amenorrhea for four months. Her skin and hair was thin and dry. Examination of the posterior oropharynx was normal. A crunching sound was heard on auscultation and palpation over the neck and upper chest. Her blood pressure was stable at $90 / 60 \mathrm{mmHg}$ and the resting heart rate was markedly reduced at 45 beats $/ \mathrm{min}$. Electrocardiogram demonstrated a sinus bradycardia.

The patient and her family reported a selfimposed caloric restriction, but no spontaneous or induced vomiting within the recent past. No other medical history was reported.

Laboratory test results were as follows: erythrocyte sedimentation rate $5 \mathrm{~mm} / \mathrm{h}$, haematocrit $38 \%$, leukocytes $3.6-4.2 \times 10^{9}$ (neu- trophils $45 \%$, lymphocytes $49 \%$, monocytes $5 \%$, eosinophils $1 \%$ ), platelets $189 \times 10^{\%} / 1$. Blood urea, serum creatinine and electrolytes were normal, while blood glucose was at low normal levels $(62 \mathrm{mmol} / \mathrm{l})$. A chest X-ray confirmed air in the mediastinum and the subcutaneous tissues of the neck and chest while findings from the lung fields were normal (figure 1A). Radiographic evaluation of the upper gastrointestinal tract after gastrografin and barium swallow showed no evidence of an oesophageal leak. Computed tomography (CT) of the neck, chest and abdomen indicated extensive subcutaneous emphysema, pneumomediastinum (with no signs of pneumothorax), retroperitoneal emphysema and epidural emphysema (air between the dura mater and the vertebral arches) and air tracking down paravertebrally through the aortic hiatus of the diaphragm down to the pelvis (figures 1B, 2A,B,C). Medical and psychiatric evaluations were consistent with a diagnosis of anorexia nervosa complicated with pneumomediastinum and soft tissue emphysema in multiple locations. No episodes of vomiting were observed during her stay in the hospital. The patient was hospitalised and confined to bed. A programme of oral nutritional supplementation combined with psychiatric behavioural therapy and cognitive psychotherapy was instituted. The patients' condition improved steadily, the interstitial air on serial chest X-rays and CT scan gradually resolved and she was discharged on the 25th day.

The patient's return to a normal diet enabled weight gain up to $54 \mathrm{~kg}$, and a chest X-ray and follow-up CT scan after two months showed total resolution of interstitial emphysema.

\section{Discussion}

Spontaneous pneumomediastinum is described as an air leak in the mediastinal tissues. This usually occurs either through oesophageal tears after vigorous vomiting, or after alveolar rupture subsequent to rapid increase of intraalveolar pressure induced by violent coughing, weight lifting, straining or emesis, ${ }^{6,7}$ with air dissecting along perivascular planes.

Vomiting is commonly self-induced by the individual with anorexia nervosa as part of the effort to lose weight. It is a recognised cause of spontaneous pneumomediastinum, although in only a few cases has it been reported as an event preceding the development of pneumomediastinum. ${ }^{7}$ In our patient vomiting did not 
precede the development of pneumomediastinum and the radiographic study of the upper gastrointestinal tract did not reveal an obvious cause for the air leak, ruling out oesophageal rupture as a possible explanation. On the other hand there were no history, symptoms, signs or radiographic evidence of bronchial asthma or pneumothorax, so that these were excluded as possible causes of increased intra-alveolar pressure. The air was presumably released from subclinical alveolar ruptures and then dissected through perivascular sheaths to the mediastinum. The precise mechanism of air tracking from the mediastinum to communicating spaces (soft tissues, neck, retroperitoneum, pelvis, spinal canal) remains unclear. It is possible that increase in mediastinal pressure allows air to dissect along the fascial planes of the great vessels, allowing air to penetrate into different locations, as has been described in cases of bronchial asthma or in pneumomediastinum developing during positive pressure ventilation..$^{5,8}$ Animal studies have demonstrated diminished alveolar elasticity following prolonged starvation. Malnourished individuals may have a similar impairment, placing them at an increased risk for alveolar rupture. ${ }^{4,7,8}$ Since known factors of increased intraalveolar pressure were absent in our case, we may assume that subclinical alveolar leaks with subsequent air dissection, pneumomediastinum and diffuse soft tissue emphysema occurred because of weakness of the alveolar wall and thinning of the connective tissue caused by severe malnutrition. ${ }^{8,9}$ Therefore, even minimal increase of the intra-alveolar pressure, such as may occur during usual daily activities, can become the cause of repeated air leaks.

Figure 1 (A) Posterioanterior chest X-ray; (B) CT scan showing extensive bilateral subcutaneous emphysema and pneumomediastinum

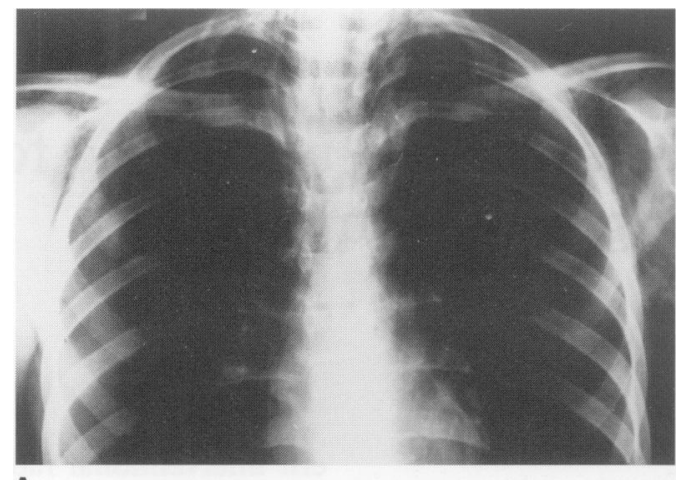

A

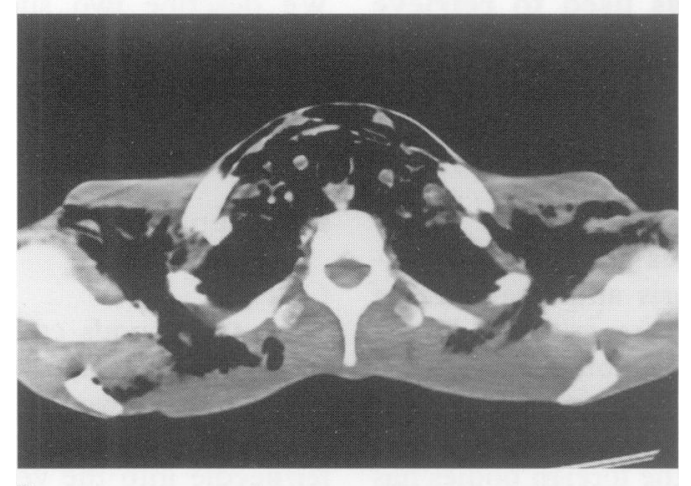

B
Thin, dry skin, although not pathognomonic, is known to have a reduced collagen content and may be a sign of the effects of starvation on connective tissue. ${ }^{10,11}$ The absence of other possible severe complications (cardiovascular, gastrointestinal, renal, haematological, skeletal, endocrine, metabolic and dermatological), described in detail by Sharp and Freeman in a recent study, may be attributed to the absence of chronicity of the disorder in our case. ${ }^{10}$ The effects of malnutrition on connective tissue may include hypoplasia, reduction or absence of building fat and gelatinous transformation, since these changes have been observed in the bone marrow of patients with anorexia nervosa and have been speculated to be due to nutritional insufficiency leading to carbohydrate and protein deficiency. ${ }^{12}$ This is confirmed indirectly in our

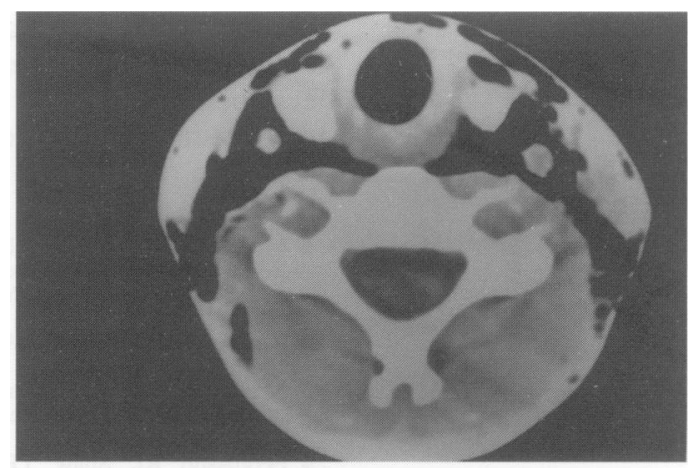

A

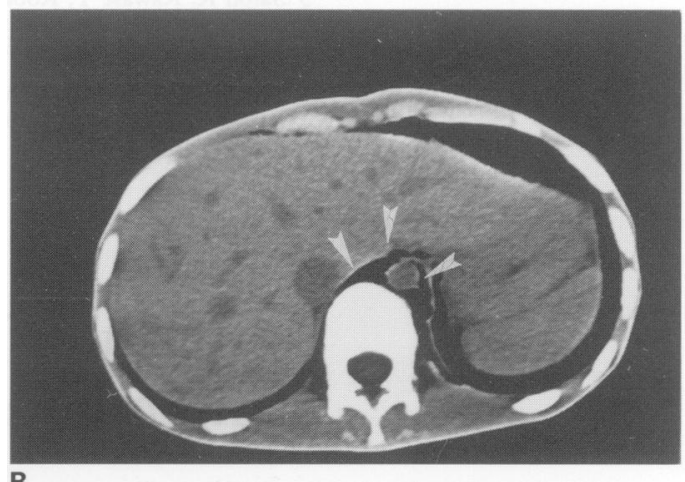
B

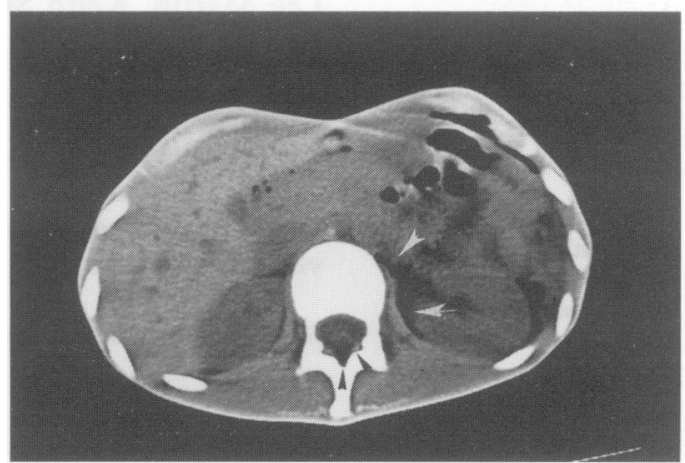

C

Figure 2 CT scans showing (A) subcutaneous emphysema at the level of the thyroid gland, (B) presence of air in the retrocrural space (white arrows), and (C) subcutaneous emphysema, retroperitoneal emphysema (white arrows) and spinal emphysema (black arrows) at the level of the kidneys 
case by the fact, that nutritional supplementation and recovery of normal body weight led to the resolution of free gas, without any other therapeutic intervention. Strict confinement to bed with restriction of activity may exert a beneficial effect, as it may prevent wide fluctuations of intra-alveolar pressure and recurrence of alveolar leaks. The extensive amount and wide distribution of free air in our patient was documented in detail, not only with radiographs, but also with CT of the neck, chest, retroperitoneal space and pelvis, and the resolution of free gas was followed up with serial CT scans.

Relative sinus bradycardia and hypotension in our patient were attributed to autonomic dysfunction which, when observed in such patients, is generally benign. However they have to be treated in order to avoid serious haemodynamic disorders, ${ }^{4}$ especially when electrolyte abnormalities due to malnutrition, vomiting or diuretic and laxative abuse coexist. ${ }^{10}$ In our case there was also a moderate leucopenia obviously due to bone marrow hypoplasia. The decrease of blood cell count

1 McAnarney ER. Physical conditions in adolescense. In: Berkow R, Fletcher AJ, eds, The Merck manual of diagnosis and therapy. Rahway, NJ: Merck \& Co, Inc, 1992; pp 227980.

2 Herzog DB, Copeland PM. Eating disorders. $N$ Engl 7 Med 1985; 313: 295-303.

3 Kaplan AS, Katz M. Eating disorders and connective tissue disease. Etiologic and treatment considerations. Psychosomatics 1992; 33: $105-8$.

4 Fergusson RJ, Shaw TR, Turnbull CM. Spontaneous pneumomediastinum: a complication of anorexia nervosa? Postgrad Med F 1985; 61: 815-7.

5 Satoh K, Kawase Y, Kobayashi T, et al. Anorexia nervosa an association with certain types of soft tissue emphysema. Nippon Kyobu Shikkan Gakkai Zashi (fapan) 1994; 32: 685-8.

6 Schulman A, Fataar S, Van Der Spui JW, et al. Air in unusual places: some causes and ramifications of pneumomediastinum. Clin Radiol 1982; 33: 301-6. may manifest as a pancytopenia, although leucopenia seems to be of greater clinical importance in patients with anorexia nervosa and must be closely monitored as it may result in increased susceptibility to infection. The diminished immunological reaction to infections without fever and leucocytosis may impede early diagnosis and lead to widespread infection in these patients. ${ }^{12}$

In conclusion it must be emphasized that the development of soft tissue emphysema in patients with anorexia nervosa is a benign condition which does not demand surgical intervention. However, because of some potentially life-threatening complications, like unexpected cardiovascular collapse, sudden death, etc, ${ }^{10}$ recognition of the primary disorder, hospitalisation and intensive nutritional rehabilitation is necessary. A complete radiographic investigation of the gastrointestinal tract is indicated in order to exclude a perforation with subsequent mediastinitis or peritonitis. A potential pitfall is that of free gas below the diaphragm that may lead to unnecessary laparotomy. ${ }^{4,7}$

7 Overby KJ, Litt IF. Mediastinal emphysema in an adolescent with anorexia nervosa and self-induced emesis. Pediatrics 1988; 81: $134-6$.

8 Satoh K, Ohkawa M, Tanabe M, Suwaki H. Anorexia nervosa with soft tissue emphysema in multiple locations (letter). AfR 1994; 163: 484.

9 Lands L, Desmond K, Demisio D, Pavilanis A, Coates AL The effects of nutritional status and hyperinflation of respiratory muscle strength in children and young adults. respiratory muscle strength in children
Am Rev Respir Dis 1990; 141: 1506-9.

Am Rev Respir Dis 1990; 141: 1506-9.
10 Sharp CW, Freeman CPL. The medical complications of Sharp CW, Freeman CPL. The medical complications

11 Savvas M, Treasure J, Studdy J, et al. The effect of anorexia nervosa on skin thickness, skin collagen and bone density Br f Obstet Gynaecol 1989; 96: 1392 - 4 .

12 Fukudo S, Tanaka A, Muranaka M, et al. Reversal of severe leukopenia by granulocyte colony-stimulating factor in anorexia nervosa. Am $\mathcal{f}$ Med Sci 1993; 305: 31 - 47 .

\section{Halton General \\ Hospital, Hospital \\ Way, Runcorn, Cheshire WA7 2DA, \\ UK \\ L Martin \\ K Strahan \\ G Murphy \\ Correspondence to: $\mathbf{M r}$ Lee Martin, Royal \\ Liverpool University \\ Hospital, Department of \\ Surgery, 8C Link, Prescot \\ Street, Liverpool L7 8XP, UK}

Accepted 9 December 1996

\title{
Retrieval of intravascular iatrogenic foreign bodies using a nonsurgical technique
}

\author{
Lee Martin, Keith Strahan, Gerry Murphy
}

\section{Summary \\ Two cases are presented in which inter- ventional radiology was used to retrieve foreign bodies from in and around the vascular system.}

Keywords: radiology, foreign bodies, catheters

Vascular access techniques are being increasingly used in the diagnosis and treatment of patients, many of whom are critically ill. As with any invasive technique, complications can occur, one of which is fracture and/or misplacement of guidewires and catheters. In the past, surgical removal of the foreign bodies has been the only option available, however mod- ern interventional radiological techniques can often obviate the need for surgical intervention. We describe two such cases, one involving removal of a knotted guidewire and the other removal of a broken guidewire fragment, both complications occurring during the routine insertion of a central venous catheter.

\section{Case reports}

\section{Case 1}

A central venous catheter was inserted, via a right subclavian approach, for venous access in a 30-year-old woman who had taken a large overdose. Initial entry of the introducer catheter/needle into the vein was difficult, necessitating several passes until good venous flow 